\title{
The existence of solutions for systems of generalized set-valued nonlinear quasi-variational inequalities
}

\author{
YQ Qiu' ${ }^{1 *}$ and XL Li
}

A correction to this article has been published:

http://www.fixedpointtheoryandapplications.com/content/2014/1/2.

"Correspondence:

qiuyangqing@sohu.com

${ }^{1}$ College of Applied Science, Jiangxi

University of Science and

Technology, Ganzhou, Jiangxi

341000 , P.R. China

Full list of author information is

available at the end of the article

\begin{abstract}
In this paper, we introduce and study a class of new systems of generalized set-valued nonlinear quasi-variational inequalities in a Hilbert space. By using the projection operator technique and the system of Wiener-Hopf equations technique, we suggest several new iterative algorithms to find the approximate solutions to these problems and prove the convergence of the different types of iterative sequences respectively. It is the first time that the system of Wiener-Hopf equations technique has been used to solve the system of variational inequalities problems, and the technique is more general than the projection operator technique. Our results improve and extend some known results in the literature.
\end{abstract}

Keywords: system of generalized set-valued nonlinear quasi-variational inequalities; projection operator technique; system of Wiener-Hopf equations; iterative algorithm; convergence criteria

\section{Introduction}

Variational inequality problems are among the most interesting and intensively studied classes of mathematics problems and have wide applications in the fields of optimization and control, economics and transportation equilibrium and engineering science. And there have been a substantial number of numerical methods including fixed point, projection operator, Wiener-Hopf equations, auxiliary principle, KKM technique, linear approximation, decomposition methods, penalty function, splitting method, inertial proximal, dynamical system and well-posedness for solving the variational inequalities and related problems in recent years (see [1-13] and the references therein).

One of the most common methods for solving the variational problem is to transfer the variational inequality into an operator equation, and then transfer the operator equation into the fixed point problems. In the present paper, we introduce and study a class of new systems of generalized set-valued nonlinear quasi-variational inequalities in a Hilbert space. We prove that the system of generalized set-valued nonlinear quasi-variational inequalities is equivalent to the fixed point problem and the system of Wiener-Hopf equations. By using the projection operator technique and the system of Wiener-Hopf equations technique, we suggest several new iterative algorithms to find the approximate so-

(C) 2013 Qiu and Li; licensee Springer. This is an Open Access article distributed under the terms of the Creative Commons Attribution License (http://creativecommons.org/licenses/by/2.0), which permits unrestricted use, distribution, and reproduction in any medium, provided the original work is properly cited. 
lutions to the problems and prove the convergence of the different types of iterative sequences. It is the first time that the system of Wiener-Hopf equations technique has been used to solve the system of variational inequalities problems, and the technique is more general than the projection operator technique. Our results improve and extend some known results in the literature.

Let $H$ be a real Hilbert space whose inner product and norm are denoted by $\langle\cdot, \cdot\rangle$ and $\|\cdot\|$ respectively. Let $K$ be a nonempty closed convex set in $H$ and $C(H)$ be the family of all nonempty compact subsets of $H$. Given two nonlinear mappings $A_{i}: K \times K \rightarrow H$ and two set-valued mappings $B_{i}: H \times H \rightarrow C(H), i=1$, 2, we consider the following problem of finding $(x, y) \in K \times K$ such that $\omega_{1} \in B_{1}(x, y), \omega_{2} \in B_{2}(x, y)$ and

$$
\begin{cases}\left\langle A_{1}(x, y)+\omega_{1}, u-x\right\rangle \geq 0, & \forall u \in K, \\ \left\langle A_{2}(x, y)+\omega_{2}, v-y\right\rangle \geq 0, & \forall v \in K,\end{cases}
$$

which is called the system of generalized set-valued nonlinear quasi-variational inequalities.

It is worth mentioning that in many important problems, the closed convex set $K$ also depends upon the solutions explicitly or implicitly. Given two point-to-set mappings $K_{1}$ : $x \rightarrow K_{1}(x)$ and $K_{2}: y \rightarrow K_{2}(y)$, which associate two closed convex sets $K_{1}(x)$ and $K_{2}(y)$ with any element $x, y$ of $H$, we consider the problem of finding $(x, y) \in K_{1}(x) \times K_{2}(y)$ such that $\omega_{1} \in B_{1}(x, y), \omega_{2} \in B_{2}(x, y)$ and

$$
\begin{cases}\left\langle A_{1}(x, y)+\omega_{1}, u-x\right\rangle \geq 0, & \forall u \in K_{1}(x), \\ \left\langle A_{2}(x, y)+\omega_{2}, v-y\right\rangle \geq 0, & \forall v \in K_{2}(y),\end{cases}
$$

which is called the system of generalized set-valued nonlinear implicit quasi-variational inequalities. We remark that if $K_{1}(x)=K_{2}(y)=K$, a nonempty closed convex set in $H$, then the problem (1.2) is exactly the problem (1.1).

If the closed convex sets $K_{1}(x)$ and $K_{2}(y)$ are of the form $K_{1}(x)=m_{1}(x)+K_{1}$ and $K_{2}(y)=$ $m_{2}(y)+K_{2}$, where $K_{1}$ and $K_{2}$ are two nonempty closed convex sets and $m_{1}, m_{2}$ are two point-to-point mappings, then the problem (1.2) is equivalent to finding $(x, y) \in K_{1}(x) \times$ $K_{2}(y)=\left(m_{1}(x)+K_{1}\right) \times\left(m_{2}(y)+K_{2}\right)$ such that $\omega_{1} \in B_{1}(x, y), \omega_{2} \in B_{2}(x, y)$ and

$$
\begin{cases}\left\langle A_{1}(x, y)+\omega_{1}, u-x\right\rangle \geq 0, & \forall u \in K_{1}(x)=m_{1}(x)+K_{1}, \\ \left\langle A_{2}(x, y)+\omega_{2}, v-y\right\rangle \geq 0, & \forall v \in K_{2}(y)=m_{2}(y)+K_{2} .\end{cases}
$$

If $A_{1}=A_{2}=V \circ A+B, B_{1}=B_{2}=T, K_{1}(x)=K_{2}(y)$, then the problem (1.2) is equivalent to finding $x \in K(x)$ such that $w \in T(x), y \in A(x)$ and

$$
\langle\omega+V y+B x, v-x\rangle \geq 0, \quad \forall v \in K(x)
$$

which is due to Noor [1].

If $A_{1}=A_{2}=A, B_{1}=B_{2}=B$, then the problem (1.1) is equivalent to finding $x \in K$ such that $w \in B(x)$ and

$$
\langle A(x)+\omega, v-x\rangle \geq 0, \quad \forall v \in K
$$




\section{Preliminaries}

We need the following known concepts and results.

Definition 2.1 (see [2-6]) Let $H$ be a Hilbert space, $A(\cdot, \cdot): H \times H \rightarrow H$ be a nonlinear mapping. $A$ is said to be

(i) $\gamma$-strongly monotone with respect to the first argument, if there exists a constant $\gamma>0$ such that

$$
\left\langle A\left(x_{1}, \cdot\right)-A\left(x_{2}, \cdot\right), x_{1}-x_{2}\right\rangle \geq \gamma\left\|x_{1}-x_{2}\right\|^{2}, \quad \forall x_{1}, x_{2} \in H
$$

Similarly, we can define $A$ is strongly monotone with respect to the second argument.

(ii) $(\tau, \zeta)$-relaxed co-coercive, if there exist constants $\tau>0, \zeta>0$ such that

$$
\left\langle A\left(x_{1}, \cdot\right)-A\left(x_{2}, \cdot\right), x_{1}-x_{2}\right\rangle \geq-\tau\left\|A\left(x_{1}, \cdot\right)-A\left(x_{2}, \cdot\right)\right\|^{2}+\zeta\left\|x_{1}-x_{2}\right\|^{2}, \quad \forall x_{1}, x_{2} \in H ;
$$

(iii) $(\xi, \eta)$-Lipschitz continuous, if there exist constants $\xi>0, \eta>0$ such that

$$
\left\|A\left(x_{1}, y_{1}\right)-A\left(x_{2}, y_{2}\right)\right\| \leq \xi\left\|x_{1}-x_{2}\right\|+\eta\left\|y_{1}-y_{2}\right\|, \quad \forall x_{1}, x_{2}, y_{1}, y_{2} \in H
$$

Definition 2.2 (see [7]) Let $B(\cdot, \cdot): H \times H \rightarrow C(H)$ be a set-valued mapping. $B$ is said to be $(\alpha, \beta)$ - $H$-Lipschitz continuous if there exist constants $\alpha>0, \beta>0$ such that

$$
H\left(B\left(x_{1}, y_{1}\right), B\left(x_{2}, y_{2}\right)\right) \leq \alpha\left\|x_{1}-x_{2}\right\|+\beta\left\|y_{1}-y_{2}\right\|, \quad \forall x_{1}, x_{2}, y_{1}, y_{2} \in H,
$$

where $H(\cdot, \cdot)$ is the Hausdorff metric on $C(H)$.

Lemma 2.3 (see[8, 9]) Let $H$ be a Hilbert space, $K$ be a nonempty closed convex set in $H$. Then, for a given $z \in H, u \in K$ satisfies the inequality

$$
\langle u-z, v-u\rangle \geq 0, \quad \forall v \in K,
$$

if and only if

$$
u=P_{K} z
$$

where $P_{K}$ is the projection of $H$ into $K$. Furthermore, the operator $P_{K}$ is nonexpansive, i.e.,

$$
\left\|P_{K}(u)-P_{K}(v)\right\| \leq\|u-v\|, \quad \forall u, v \in H .
$$

Assumption 2.4 Let $H$ be a real Hilbert space, $K_{1}(x)$ and $K_{2}(y)$ be two nonempty closed convex sets. For all $x, y, z \in H$, the operators $P_{K_{1}(x)}$ and $P_{K_{2}(y)}$ satisfy the relations

$$
\begin{gathered}
\left\|P_{K_{1}(x)} z-P_{K_{1}(y)} z\right\| \leq s_{1}\|x-y\|, \\
\left\|P_{K_{2}(x)} z-P_{K_{2}(y)} z\right\| \leq s_{2}\|x-y\|,
\end{gathered}
$$

where $s_{1}>0, s_{2}>0$ are two constants. 
Remark 2.5 We remark that Assumption 2.4 is also true for the case $K_{1}(x)=m_{1}(x)+K_{1}$, $K_{2}(y)=m_{2}(y)+K_{2}$, when the point-to-point mappings $m_{1}, m_{2}$ are $\mu_{1}, \mu_{2}$-Lipschitz continuous respectively. For all $x, y, z \in H$, it is well known that

$$
\begin{aligned}
& P_{K_{1}(x)} z=P_{m_{1}(x)+K_{1}} z=m_{1}(x)+P_{K_{1}}\left[z-m_{1}(x)\right], \\
& P_{K_{2}(y)} z=P_{m_{2}(y)+K_{2}} z=m_{2}(y)+P_{K_{2}}\left[z-m_{2}(y)\right],
\end{aligned}
$$

and

$$
\begin{aligned}
\left\|P_{K_{1}(x)} z-P_{K_{1}(y)} z\right\| & =\left\|m_{1}(x)-m_{1}(y)+P_{K_{1}}\left[z-m_{1}(x)\right]-P_{K_{1}}\left[z-m_{1}(y)\right]\right\| \\
& \leq\left\|m_{1}(x)-m_{1}(y)\right\|+\left\|P_{K_{1}}\left[z-m_{1}(x)\right]-P_{K_{1}}\left[z-m_{1}(y)\right]\right\| \\
& \leq 2\left\|m_{1}(x)-m_{1}(y)\right\| \leq 2 \mu_{1}\|x-y\|,
\end{aligned}
$$

which shows that (2.1) holds for $s_{1}=2 \mu_{1}>0$. Similarly, (2.2) holds for $s_{2}=2 \mu_{2}>0$.

Lemma 2.6 The system of generalized set-valued nonlinear implicit quasi-variational inequalities $(1.2)$ has solutions $\left(x, y, \omega_{1}, \omega_{2}\right)$ if and only if $\left(x, y, \omega_{1}, \omega_{2}\right)$ satisfy the relations

$$
\left\{\begin{array}{l}
x=P_{K_{1}(x)}\left[x-\rho_{1}\left(A_{1}(x, y)+\omega_{1}\right)\right], \\
y=P_{K_{2}(y)}\left[y-\rho_{2}\left(A_{2}(x, y)+\omega_{2}\right)\right],
\end{array}\right.
$$

where $(x, y) \in K_{1}(x) \times K_{2}(y), \omega_{1} \in B_{1}(x, y), \omega_{2} \in B_{2}(x, y), \rho_{1}, \rho_{2}>0$ are two constants. $P_{K_{1}(x)}$ : $H \rightarrow K_{1}(x), P_{K_{2}(y)}: H \rightarrow K_{2}(y)$ are two projection operators.

Proof The conclusion follows directly from Lemma 2.3 .

Lemma 2.7 (see[10,11]) Let $E$ be a complete metric space, $C B(E)$ be the family of all the convex bounded subsets of $E, T: E \rightarrow C B(E)$ be a set-valued mapping. Then for any given $\varepsilon>0$ and any given $x, y \in E, u \in T x$, there exists $v \in$ Ty such that

$$
d(u, v) \leq(1+\varepsilon) H(T x, T y)
$$

where $H(\cdot, \cdot)$ is the Hausdorff metric on $C B(E)$.

\section{Projection operator technique}

Using the projection operator technique, Lemma 2.6 and Lemma 2.7, we construct the following iterative algorithms.

Algorithm 3.1 Let $H$ be a real Hilbert space, $K_{1}(x)$ and $K_{2}(y)$ be two nonempty closed convex sets in $H, A_{i}: K_{1}(x) \times K_{2}(y) \rightarrow H$ be two nonlinear mappings, $B_{i}: H \times H \rightarrow C(H)$ be two set-valued mappings, $i=1,2$. For any given $\left(x_{0}, y_{0}\right) \in K_{1}\left(x_{0}\right) \times K_{2}\left(y_{0}\right)$ such that $\omega_{0}^{1} \in B_{1}\left(x_{0}, y_{0}\right), \omega_{0}^{2} \in B_{2}\left(x_{0}, y_{0}\right)$, and

$$
\begin{aligned}
& x_{1}=\left(1-\lambda_{1}\right) x_{0}+\lambda_{1} P_{K_{1}\left(x_{0}\right)}\left[x_{0}-\rho_{1}\left(A_{1}\left(x_{0}, y_{0}\right)+\omega_{0}^{1}\right)\right], \\
& y_{1}=\left(1-\lambda_{2}\right) y_{0}+\lambda_{2} P_{K_{2}\left(y_{0}\right)}\left[y_{0}-\rho_{2}\left(A_{2}\left(x_{0}, y_{0}\right)+\omega_{0}^{2}\right)\right] .
\end{aligned}
$$


Since $\omega_{0}^{1} \in B_{1}\left(x_{0}, y_{0}\right), \omega_{0}^{2} \in B_{2}\left(x_{0}, y_{0}\right)$, and by Lemma 2.7, there exist $\omega_{1}^{1} \in B_{1}\left(x_{1}, y_{1}\right), \omega_{1}^{2} \in$ $B_{2}\left(x_{1}, y_{1}\right)$ such that

$$
\begin{aligned}
\left\|\omega_{1}^{1}-\omega_{0}^{1}\right\| & \leq(1+1) H\left(B_{1}\left(x_{1}, y_{1}\right), B_{1}\left(x_{0}, y_{0}\right)\right), \\
\left\|\omega_{1}^{2}-\omega_{0}^{2}\right\| & \leq(1+1) H\left(B_{2}\left(x_{1}, y_{1}\right), B_{2}\left(x_{0}, y_{0}\right)\right) .
\end{aligned}
$$

Let

$$
\begin{aligned}
& x_{2}=\left(1-\lambda_{1}\right) x_{1}+\lambda_{1} P_{K_{1}\left(x_{1}\right)}\left[x_{1}-\rho_{1}\left(A_{1}\left(x_{1}, y_{1}\right)+\omega_{1}^{1}\right)\right], \\
& y_{2}=\left(1-\lambda_{2}\right) y_{1}+\lambda_{2} P_{K_{2}\left(y_{1}\right)}\left[y_{1}-\rho_{2}\left(A_{2}\left(x_{1}, y_{1}\right)+\omega_{1}^{2}\right)\right],
\end{aligned}
$$

since $\omega_{1}^{1} \in B_{1}\left(x_{1}, y_{1}\right), \omega_{1}^{2} \in B_{2}\left(x_{1}, y_{1}\right)$, and by Lemma 2.7 , there exist $\omega_{2}^{1} \in B_{1}\left(x_{2}, y_{2}\right), \omega_{2}^{2} \in$ $B_{2}\left(x_{2}, y_{2}\right)$ such that

$$
\begin{aligned}
\left\|\omega_{2}^{1}-\omega_{1}^{1}\right\| & \leq\left(1+\frac{1}{2}\right) H\left(B_{1}\left(x_{2}, y_{2}\right), B_{1}\left(x_{1}, y_{1}\right)\right), \\
\left\|\omega_{2}^{2}-\omega_{1}^{2}\right\| & \leq\left(1+\frac{1}{2}\right) H\left(B_{2}\left(x_{2}, y_{2}\right), B_{2}\left(x_{1}, y_{1}\right)\right) .
\end{aligned}
$$

By induction, we can define iterative sequences $\left\{x_{n}\right\},\left\{y_{n}\right\},\left\{\omega_{n}^{1}\right\}$ and $\left\{\omega_{n}^{2}\right\}$ satisfying

$$
\begin{aligned}
& x_{n+1}=\left(1-\lambda_{1}\right) x_{n}+\lambda_{1} P_{K_{1}\left(x_{n}\right)}\left[x_{n}-\rho_{1}\left(A_{1}\left(x_{n}, y_{n}\right)+\omega_{n}^{1}\right)\right], \\
& y_{n+1}=\left(1-\lambda_{2}\right) y_{n}+\lambda_{2} P_{K_{2}\left(y_{n}\right)}\left[y_{n}-\rho_{2}\left(A_{2}\left(x_{n}, y_{n}\right)+\omega_{n}^{2}\right)\right], \\
& \omega_{n}^{1} \in B_{1}\left(x_{n}, y_{n}\right), \quad\left\|\omega_{n+1}^{1}-\omega_{n}^{1}\right\| \leq\left(1+\frac{1}{n+1}\right) H\left(B_{1}\left(x_{n+1}, y_{n+1}\right), B_{1}\left(x_{n}, y_{n}\right)\right), \\
& \omega_{n}^{2} \in B_{2}\left(x_{n}, y_{n}\right), \quad\left\|\omega_{n+1}^{2}-\omega_{n}^{2}\right\| \leq\left(1+\frac{1}{n+1}\right) H\left(B_{2}\left(x_{n+1}, y_{n+1}\right), B_{2}\left(x_{n}, y_{n}\right)\right),
\end{aligned}
$$

where $n=0,1,2, \ldots$

If $K_{1}(x)=K_{2}(y)=K$, we obtain the following algorithm from Algorithm 3.1.

Algorithm 3.2 We define iterative sequences $\left\{x_{n}\right\},\left\{y_{n}\right\},\left\{\omega_{n}^{1}\right\}$ and $\left\{\omega_{n}^{2}\right\}$ satisfying

$$
\begin{aligned}
& x_{n+1}=\left(1-\lambda_{1}\right) x_{n}+\lambda_{1} P_{K}\left[x_{n}-\rho_{1}\left(A_{1}\left(x_{n}, y_{n}\right)+\omega_{n}^{1}\right)\right], \\
& y_{n+1}=\left(1-\lambda_{2}\right) y_{n}+\lambda_{2} P_{K}\left[y_{n}-\rho_{2}\left(A_{2}\left(x_{n}, y_{n}\right)+\omega_{n}^{2}\right)\right], \\
& \omega_{n}^{1} \in B_{1}\left(x_{n}, y_{n}\right), \quad\left\|\omega_{n+1}^{1}-\omega_{n}^{1}\right\| \leq\left(1+\frac{1}{n+1}\right) H\left(B_{1}\left(x_{n+1}, y_{n+1}\right), B_{1}\left(x_{n}, y_{n}\right)\right), \\
& \omega_{n}^{2} \in B_{2}\left(x_{n}, y_{n}\right), \quad\left\|\omega_{n+1}^{2}-\omega_{n}^{2}\right\| \leq\left(1+\frac{1}{n+1}\right) H\left(B_{2}\left(x_{n+1}, y_{n+1}\right), B_{2}\left(x_{n}, y_{n}\right)\right),
\end{aligned}
$$

where $n=0,1,2, \ldots$

Theorem 3.3 Let $H$ be a real Hilbert space, $K_{1}(x)$ and $K_{2}(y)$ be two nonempty closed convex sets in $H$. For $i=1,2$, let nonlinear mappings $A_{i}: K \times K \rightarrow H$ be $\left(\xi_{i}, \eta_{i}\right)$-Lipschitz continuous and $\gamma_{i}$-strongly monotone with respect to the ith argument, $B_{i}: H \times H \rightarrow C(H)$ be $\left(\alpha_{i}, \beta_{i}\right)$ - 
H-Lipschitz continuous if Assumption 2.4 holds, and there exist constants $\rho_{i}>0,0<\lambda_{i}<1$ such that

$$
\begin{aligned}
0< & \max \left\{1-\lambda_{1}\left[1-\left(s_{1}+\sqrt{1-2 \rho_{1} \gamma_{1}+\rho_{1}^{2} \xi_{1}^{2}}+\rho_{1} \alpha_{1}\right)\right]+\lambda_{2} \rho_{2}\left(\alpha_{2}+\xi_{2}\right),\right. \\
& \left.1-\lambda_{2}\left[1-\left(s_{2}+\sqrt{1-2 \rho_{2} \gamma_{2}+\rho_{2}^{2} \eta_{2}^{2}}+\rho_{2} \beta_{2}\right)\right]+\lambda_{1} \rho_{1}\left(\beta_{1}+\eta_{1}\right)\right\}<1,
\end{aligned}
$$

then the problem (1.2) admits solutions $\left(x, y, \omega_{1}, \omega_{2}\right)$ and sequences $\left\{x_{n}\right\},\left\{y_{n}\right\},\left\{\omega_{n}^{1}\right\}$ and $\left\{\omega_{n}^{2}\right\}$ which are generated by Algorithm 3.1 converge to $x, y, \omega_{1}$ and $\omega_{2}$ respectively.

Proof By Lemma 2.3, (2.1) and (3.1), we have

$$
\begin{aligned}
\| x_{n+1} & -x_{n} \| \\
= & \|\left(1-\lambda_{1}\right) x_{n}+\lambda_{1} P_{K_{1}\left(x_{n}\right)}\left[x_{n}-\rho_{1}\left(A_{1}\left(x_{n}, y_{n}\right)+\omega_{n}^{1}\right)\right] \\
& -\left(1-\lambda_{1}\right) x_{n-1}-\lambda_{1} P_{K_{1}\left(x_{n-1}\right)}\left[x_{n-1}-\rho_{1}\left(A_{1}\left(x_{n-1}, y_{n-1}\right)+\omega_{n-1}^{1}\right)\right] \| \\
\leq & \left(1-\lambda_{1}\right)\left\|x_{n}-x_{n-1}\right\| \\
& +\lambda_{1}\left\|P_{K_{1}\left(x_{n}\right)}\left[x_{n}-\rho_{1}\left(A_{1}\left(x_{n}, y_{n}\right)+\omega_{n}^{1}\right)\right]-P_{K_{1}\left(x_{n-1}\right)}\left[x_{n}-\rho_{1}\left(A_{1}\left(x_{n}, y_{n}\right)+\omega_{n}^{1}\right)\right]\right\| \\
& +\lambda_{1} \| P_{K_{1}\left(x_{n-1}\right)}\left[x_{n}-\rho_{1}\left(A_{1}\left(x_{n}, y_{n}\right)+\omega_{n}^{1}\right)\right] \\
& -P_{K_{1}\left(x_{n-1}\right)}\left[x_{n-1}-\rho_{1}\left(A_{1}\left(x_{n-1}, y_{n-1}\right)+\omega_{n-1}^{1}\right)\right] \| \\
\leq & \left(1-\lambda_{1}+\lambda_{1} s_{1}\right)\left\|x_{n}-x_{n-1}\right\| \\
& +\lambda_{1}\left\|\left[x_{n}-\rho_{1}\left(A_{1}\left(x_{n}, y_{n}\right)+\omega_{n}^{1}\right)\right]-\left[x_{n-1}-\rho_{1}\left(A_{1}\left(x_{n-1}, y_{n-1}\right)+\omega_{n-1}^{1}\right)\right]\right\| \\
\leq & \left(1-\lambda_{1}+\lambda_{1} s_{1}\right)\left\|x_{n}-x_{n-1}\right\|+\lambda_{1}\left\|x_{n}-x_{n-1}-\rho_{1}\left(A_{1}\left(x_{n}, y_{n}\right)-A_{1}\left(x_{n-1}, y_{n}\right)\right)\right\| \\
& +\lambda_{1} \rho_{1}\left[\left\|\omega_{n}^{1}-\omega_{n-1}^{1}\right\|+\left\|A_{1}\left(x_{n-1}, y_{n}\right)-A_{1}\left(x_{n-1}, y_{n-1}\right)\right\|\right] .
\end{aligned}
$$

Since $A_{1}$ is $\gamma_{1}$-strongly monotone with respect to the first argument and Lipschitz continuous, we have

$$
\begin{aligned}
& \left\|x_{n}-x_{n-1}-\rho_{1}\left(A_{1}\left(x_{n}, y_{n}\right)-A_{1}\left(x_{n-1}, y_{n}\right)\right)\right\|^{2} \\
& =\left\|x_{n}-x_{n-1}\right\|^{2}-2 \rho_{1}\left\langle A_{1}\left(x_{n}, y_{n}\right)-A_{1}\left(x_{n-1}, y_{n}\right), x_{n}-x_{n-1}\right\rangle \\
& \quad+\rho_{1}^{2}\left\|A_{1}\left(x_{n}, y_{n}\right)-A_{1}\left(x_{n-1}, y_{n}\right)\right\|^{2} \\
& \leq\left(1-2 \rho_{1} \gamma_{1}+\rho_{1}^{2} \xi_{1}^{2}\right)\left\|x_{n}-x_{n-1}\right\|^{2},
\end{aligned}
$$

and

$$
\left\|A_{1}\left(x_{n-1}, y_{n}\right)-A_{1}\left(x_{n-1}, y_{n-1}\right)\right\| \leq \eta_{1}\left\|y_{n}-y_{n-1}\right\| .
$$

By (3.3) and $\left(\alpha_{1}, \beta_{1}\right)-H$-Lipschitz continuity of $B_{1}$, we have

$$
\begin{aligned}
\left\|\omega_{n}^{1}-\omega_{n-1}^{1}\right\| & \leq\left(1+\frac{1}{n}\right) H\left(B_{1}\left(x_{n}, y_{n}\right), B_{1}\left(x_{n-1}, y_{n-1}\right)\right), \\
& \leq\left(1+\frac{1}{n}\right)\left[\alpha_{1}\left\|x_{n}-x_{n-1}\right\|+\beta_{1}\left\|y_{n}-y_{n-1}\right\|\right] .
\end{aligned}
$$


Combining (3.6), (3.7), (3.8) and (3.9), we obtain

$$
\begin{aligned}
\left\|x_{n+1}-x_{n}\right\| \leq & \left\{1-\lambda_{1}\left[1-\left(s_{1}+\sqrt{1-2 \rho_{1} \gamma_{1}+\rho_{1}^{2} \xi_{1}^{2}}+\rho_{1}\left(1+\frac{1}{n}\right) \alpha_{1}\right)\right]\right\}\left\|x_{n}-x_{n-1}\right\| \\
& +\lambda_{1} \rho_{1}\left[\left(1+\frac{1}{n}\right) \beta_{1}+\eta_{1}\right]\left\|y_{n}-y_{n-1}\right\| .
\end{aligned}
$$

Similarly, we can have

$$
\begin{aligned}
\left\|y_{n+1}-y_{n}\right\| \leq & \left\{1-\lambda_{2}\left[1-\left(s_{2}+\sqrt{1-2 \rho_{2} \gamma_{2}+\rho_{2}^{2} \eta_{2}^{2}}+\rho_{2}\left(1+\frac{1}{n}\right) \beta_{2}\right)\right]\right\}\left\|y_{n}-y_{n-1}\right\| \\
& +\lambda_{2} \rho_{2}\left[\left(1+\frac{1}{n}\right) \alpha_{2}+\xi_{2}\right]\left\|x_{n}-x_{n-1}\right\| .
\end{aligned}
$$

Adding (3.10) to (3.11), we have

$$
\begin{aligned}
& \left\|x_{n+1}-x_{n}\right\|+\left\|y_{n+1}-y_{n}\right\| \\
& \leq\left\{1-\lambda_{1}\left[1-\left(s_{1}+\sqrt{1-2 \rho_{1} \gamma_{1}+\rho_{1}^{2} \xi_{1}^{2}}+\rho_{1}\left(1+\frac{1}{n}\right) \alpha_{1}\right)\right]\right. \\
& \left.\quad+\lambda_{2} \rho_{2}\left[\left(1+\frac{1}{n}\right) \alpha_{2}+\xi_{2}\right]\right\}\left\|x_{n}-x_{n-1}\right\| \\
& \quad+\left\{1-\lambda_{2}\left[1-\left(s_{2}+\sqrt{1-2 \rho_{2} \gamma_{2}+\rho_{2}^{2} \eta_{2}^{2}}+\rho_{2}\left(1+\frac{1}{n}\right) \beta_{2}\right)\right]\right. \\
& \left.\quad+\lambda_{1} \rho_{1}\left[\left(1+\frac{1}{n}\right) \beta_{1}+\eta_{1}\right]\right\}\left\|y_{n}-y_{n-1}\right\| \\
& \leq \theta_{n}\left(\left\|x_{n}-x_{n-1}\right\|+\left\|y_{n}-y_{n-1}\right\|\right),
\end{aligned}
$$

where

$$
\begin{aligned}
\theta_{n}= & \max \left\{1-\lambda_{1}\left[1-\left(s_{1}+\sqrt{1-2 \rho_{1} \gamma_{1}+\rho_{1}^{2} \xi_{1}^{2}}+\rho_{1}\left(1+\frac{1}{n}\right) \alpha_{1}\right)\right]\right. \\
& +\lambda_{2} \rho_{2}\left[\left(1+\frac{1}{n}\right) \alpha_{2}+\xi_{2}\right], 1-\lambda_{2}\left[1-\left(s_{2}+\sqrt{1-2 \rho_{2} \gamma_{2}+\rho_{2}^{2} \eta_{2}^{2}}+\rho_{2}\left(1+\frac{1}{n}\right) \beta_{2}\right)\right] \\
& \left.+\lambda_{1} \rho_{1}\left[\left(1+\frac{1}{n}\right) \beta_{1}+\eta_{1}\right]\right\} .
\end{aligned}
$$

Let

$$
\begin{aligned}
\theta= & \max \left\{1-\lambda_{1}\left[1-\left(s_{1}+\sqrt{1-2 \rho_{1} \gamma_{1}+\rho_{1}^{2} \xi_{1}^{2}}+\rho_{1} \alpha_{1}\right)\right]+\lambda_{2} \rho_{2}\left(\alpha_{2}+\xi_{2}\right),\right. \\
& \left.1-\lambda_{2}\left[1-\left(s_{2}+\sqrt{1-2 \rho_{2} \gamma_{2}+\rho_{2}^{2} \eta_{2}^{2}}+\rho_{2} \beta_{2}\right)\right]+\lambda_{1} \rho_{1}\left(\beta_{1}+\eta_{1}\right)\right\}
\end{aligned}
$$

then $\theta_{n} \rightarrow \theta$ as $n \rightarrow \infty$. By (3.5), we know that $0<\theta<1$. So, (3.12) implies that $\left\{x_{n}\right\}$ and $\left\{y_{n}\right\}$ are both Cauchy sequences. Thus, there exist $x \in K_{1}(x)$ and $y \in K_{2}(y)$ such that $x_{n} \rightarrow x$, $y_{n} \rightarrow y$ as $n \rightarrow \infty$. 
Now, we prove that $\omega_{n}^{1} \rightarrow \omega_{1} \in B_{1}(x, y)$ and $\omega_{n}^{2} \rightarrow \omega_{2} \in B_{2}(x, y)$. In fact, since $\left\{x_{n}\right\}$ and $\left\{y_{n}\right\}$ are both Cauchy sequences and by (3.9), we know that $\left\{\omega_{n}^{1}\right\}$ is Cauchy sequences. Similarly, $\left\{\omega_{n}^{2}\right\}$ is also Cauchy sequences. Therefore, there exist $\omega_{1} \in C(H)$ and $\omega_{2} \in C(H)$ such that $\omega_{n}^{1} \rightarrow \omega_{1}$ and $\omega_{n}^{2} \rightarrow \omega_{2}$. Further,

$$
\begin{aligned}
d\left(\omega_{1}, B_{1}(x, y)\right) & \leq\left\|\omega_{1}-\omega_{n}^{1}\right\|+d\left(\omega_{n}^{1}, B_{1}(x, y)\right) \\
& \leq\left\|\omega_{1}-\omega_{n}^{1}\right\|+H\left(B_{1}\left(x_{n}, y_{n}\right), B_{1}(x, y)\right) \\
& \leq\left\|\omega_{1}-\omega_{n}^{1}\right\|+\alpha_{1}\left\|x_{n}-x\right\|+\beta_{1}\left\|y_{n}-y\right\| \rightarrow 0 \quad(n \rightarrow \infty) .
\end{aligned}
$$

Since $B_{1}(x, y)$ is compact, we have $\omega_{1} \in B_{1}(x, y)$. Similarly, we have $\omega_{2} \in B_{2}(x, y)$.

By the continuity of $A_{1}, A_{2}, B_{1}, B_{2}, P_{K_{1}(x)}, P_{K_{2}(y)}$ and Algorithm 3.1, we know that $\left(x, y, \omega_{1}, \omega_{2}\right)$ satisfy the relations (2.3). By Lemma 2.6 , we claim that $\left(x, y, \omega_{1}, \omega_{2}\right)$ is a solution of the problem (1.2). This completes the proof.

If $K_{1}(x)=K_{2}(y)=K$, we do not need Assumption 2.4 and can obtain the following theorem from Theorem 3.3.

Theorem 3.4 Let $H$ be a real Hilbert space, $K$ be a nonempty closed convex set in $H$. For $i=1,2$, let nonlinear mappings $A_{i}: K \times K \rightarrow H$ be $\left(\xi_{i}, \eta_{i}\right)$-Lipschitz continuous and $\gamma_{i}$-strongly monotone with respect to the ith argument, $B_{i}: H \times H \rightarrow C(H)$ be $\left(\alpha_{i}, \beta_{i}\right)$ - $H$ Lipschitz continuous, if there exist constants $\rho_{i}>0,0<\lambda_{i}<1$ such that

$$
\begin{aligned}
0< & \max \left\{1-\lambda_{1}\left[1-\left(\sqrt{1-2 \rho_{1} \gamma_{1}+\rho_{1}^{2} \xi_{1}^{2}}+\rho_{1} \alpha_{1}\right)\right]+\lambda_{2} \rho_{2}\left(\alpha_{2}+\xi_{2}\right),\right. \\
& \left.1-\lambda_{2}\left[1-\left(\sqrt{1-2 \rho_{2} \gamma_{2}+\rho_{2}^{2} \eta_{2}^{2}}+\rho_{2} \beta_{2}\right)\right]+\lambda_{1} \rho_{1}\left(\beta_{1}+\eta_{1}\right)\right\}<1,
\end{aligned}
$$

then the problem (1.1) admits solutions $\left(x, y, \omega_{1}, \omega_{2}\right)$ and sequences $\left\{x_{n}\right\},\left\{y_{n}\right\},\left\{\omega_{n}^{1}\right\}$ and $\left\{\omega_{n}^{2}\right\}$ which are generated by Algorithm 3.2 converge to $x, y, \omega_{1}$ and $\omega_{2}$ respectively.

\section{System of Wiener-Hopf equations technique}

Related to the system of generalized set-valued nonlinear implicit quasi-variational inequalities (1.2), we now consider a new system of generalized implicit Wiener-Hopf equations (4.1). And we will establish the equivalence between them. This equivalence is then used to suggest a number of new iterative algorithms for solving the given systems of variational inequalities.

To be more precise, let $Q_{K_{1}(x)}=I-P_{K_{1}(x)}, Q_{K_{2}(y)}=I-P_{K_{2}(y)}$, where $I$ is the identity operator, $P_{K_{1}(x)}: H \rightarrow K_{1}(x)$ and $P_{K_{2}(y)}: H \rightarrow K_{2}(y)$ are two projection operators, $K_{1}(x)$ and $K_{2}(y)$ are two convex sets. We consider the following problem of finding $(x, y) \in K_{1}(x) \times K_{2}(y)$, $\left(z_{1}, z_{2}\right) \in H \times H$ such that $\omega_{1} \in B_{1}\left(P_{K_{1}(x)} z_{1}, P_{K_{2}(y)} z_{2}\right), \omega_{2} \in B_{2}\left(P_{K_{1}(x)} z_{1}, P_{K_{2}(y)} z_{2}\right)$ and

$$
\left\{\begin{array}{l}
A_{1}\left(P_{K_{1}(x)} z_{1}, P_{K_{2}(y)} z_{2}\right)+\omega_{1}+\rho_{1}^{-1} Q_{K_{1}(x)} z_{1}=0 \\
A_{2}\left(P_{K_{1}(x)} z_{1}, P_{K_{2}(y)} z_{2}\right)+\omega_{2}+\rho_{2}^{-1} Q_{K_{2}(y)} z_{2}=0
\end{array}\right.
$$

where $\rho_{1}>0, \rho_{2}>0$ are constants. (4.1) is called the system of generalized implicit WienerHopf equations. 
If $K_{1}(x)=K_{2}(y)=K$, we obtain the following system of generalized Wiener-Hopf equations from (4.1), which is of finding $\left(z_{1}, z_{2}\right) \in H \times H$ such that $\omega_{1} \in B_{1}\left(P_{K} z_{1}, P_{K} z_{2}\right), \omega_{2} \in$ $B_{2}\left(P_{K} z_{1}, P_{K} z_{2}\right)$ and

$$
\left\{\begin{array}{l}
A_{1}\left(P_{K} z_{1}, P_{K} z_{2}\right)+\omega_{1}+\rho_{1}^{-1} Q_{K} z_{1}=0 \\
A_{2}\left(P_{K} z_{1}, P_{K} z_{2}\right)+\omega_{2}+\rho_{2}^{-1} Q_{K} z_{2}=0
\end{array}\right.
$$

where $\rho_{1}>0, \rho_{2}>0$ are constants.

If $A_{1}=A_{2}=A, B_{1}=B_{2}=B$, we obtain the following Wiener-Hopf equation from (4.2), which is of finding $z \in H$ such that $\omega \in B\left(P_{K} z\right)$ and

$$
A\left(P_{K} z\right)+\omega+\rho^{-1} Q_{K} z=0
$$

where $\rho>0$ is a constant.

Lemma 4.1 The system of generalized set-valued nonlinear implicit quasi-variational inequalities (1.2) has solutions $(x, y) \in K_{1}(x) \times K_{2}(y)$ such that $\omega_{1} \in B_{1}(x, y), \omega_{2} \in B_{2}(x, y)$ if and only if the system of generalized implicit Wiener-Hopf equations (4.1) has solutions $(x, y) \in K_{1}(x) \times K_{2}(y)$ and $\left(z_{1}, z_{2}\right) \in H \times H$ such that $\omega_{1} \in B_{1}(x, y), \omega_{2} \in B_{2}(x, y)$, where

$$
\left\{\begin{array}{l}
x=P_{K_{1}(x)} z_{1} \\
y=P_{K_{2}(y)} z_{2} \\
z_{1}=x-\rho_{1}\left(A_{1}(x, y)+\omega_{1}\right), \\
z_{2}=y-\rho_{2}\left(A_{2}(x, y)+\omega_{2}\right),
\end{array}\right.
$$

and $\rho_{1}>0, \rho_{2}>0$ are constants.

Proof Let $(x, y) \in K_{1}(x) \times K_{2}(y)$ such that $\omega_{1} \in B_{1}(x, y), \omega_{2} \in B_{2}(x, y)$ be a solution of (1.2), then by Lemma 2.6, we know that $(x, y)$ satisfy $(2.3)$.

Let $z_{1}=x-\rho_{1}\left(A_{1}(x, y)+\omega_{1}\right), z_{2}=y-\rho_{2}\left(A_{2}(x, y)+\omega_{2}\right)$, then by $(2.3)$, we have $x=P_{K_{1}(x)} z_{1}$, $y=P_{K_{2}(y)} z_{2}$, which is just (4.4). And we have

$$
\left\{\begin{array}{l}
z_{1}=P_{K_{1}(x)} z_{1}-\rho_{1}\left(A_{1}(x, y)+\omega_{1}\right) \\
z_{2}=P_{K_{2}(y)} z_{2}-\rho_{2}\left(A_{2}(x, y)+\omega_{2}\right)
\end{array}\right.
$$

Using the fact $Q_{K_{1}(x)}=I-P_{K_{1}(x)}$ and $Q_{K_{2}(y)}=I-P_{K_{2}(y)}$, we obtain (4.1). That is to say, $(x, y) \in K_{1}(x) \times K_{2}(y)$ and $\left(z_{1}, z_{2}\right) \in H \times H$ such that $\omega_{1} \in B_{1}\left(P_{K_{1}(x)} z_{1}, P_{K_{2}(y)} z_{2}\right), \omega_{2} \in$ $B_{2}\left(P_{K_{1}(x)} z_{1}, P_{K_{2}(y)} z_{2}\right)$ is also the solution of (4.1).

Conversely, let $(x, y) \in K_{1}(x) \times K_{2}(y)$ and $\left(z_{1}, z_{2}\right) \in H \times H$ such that $\omega_{1} \in B_{1}(x, y), \omega_{2} \in$ $B_{2}(x, y)$ be a solution of (4.1). Then we have

$$
\left\{\begin{array}{l}
\rho_{1}\left(A_{1}\left(P_{K_{1}(x)} z_{1}, P_{K_{2}(y)} z_{2}\right)+\omega_{1}\right)+z_{1}=P_{K_{1}(x)} z_{1} \\
\rho_{2}\left(A_{2}\left(P_{K_{1}(x)} z_{1}, P_{K_{2}(y)} z_{2}\right)+\omega_{2}\right)+z_{2}=P_{K_{2}(y)} z_{2}
\end{array}\right.
$$


Now, by invoking Lemma 2.3 and the above relations, we have

$$
\begin{cases}0 \leq\left\langle P_{K_{1}(x)} z_{1}-z_{1}, u-P_{K_{1}(x)} z_{1}\right\rangle, & \forall u \in K_{1}(x), \\ 0 \leq\left\langle P_{K_{2}(y)} z_{2}-z_{2}, v-P_{K_{2}(y)} z_{2}\right\rangle, & \forall v \in K_{2}(y) .\end{cases}
$$

Thus $\left(x, y, \omega_{1}, \omega_{2}\right)$, where

$$
\left\{\begin{array}{l}
x=P_{K_{1}(x)} z_{1}, \\
y=P_{K_{2}(y)} z_{2},
\end{array}\right.
$$

is a solution of (1.2).

If $K_{1}(x)=K_{2}(y)=K$, we obtain the following lemma from Lemma 4.1.

Lemma 4.2 The system of generalized set-valued nonlinear quasi-variational inequalities (1.1) has solutions $(x, y) \in K \times K$ such that $\omega_{1} \in B_{1}(x, y), \omega_{2} \in B_{2}(x, y)$ if and only if the system of generalized Wiener-Hopf equations (4.2) has solutions $(x, y) \in K \times K$ and $\left(z_{1}, z_{2}\right) \in H \times H$ such that $\omega_{1} \in B_{1}(x, y), \omega_{2} \in B_{2}(x, y)$, where

$$
\left\{\begin{array}{l}
x=P_{K} z_{1} \\
y=P_{K} z_{2} \\
z_{1}=x-\rho_{1}\left(A_{1}(x, y)+\omega_{1}\right) \\
z_{2}=y-\rho_{2}\left(A_{2}(x, y)+\omega_{2}\right)
\end{array}\right.
$$

and $\rho_{1}>0, \rho_{2}>0$ are constants.

Using the system of Wiener-Hopf equations technique, Lemma 4.1 and Lemma 2.7, we construct the following iterative algorithms.

Algorithm 4.3 Let $H$ be a real Hilbert space, $K_{1}(x)$ and $K_{2}(y)$ be two nonempty closed convex sets in $H, A_{i}: K_{1}(x) \times K_{2}(y) \rightarrow H$ be two nonlinear mappings, $B_{i}: H \times H \rightarrow$ $C(H)$ be two set-valued mappings, $i=1,2$. For any given $\left(z_{0}^{1}, z_{0}^{2}\right) \in H \times H$, such that $x_{0}=P_{K_{1}\left(x_{0}\right)} z_{0}^{1} \in K_{1}\left(x_{0}\right), y_{0}=P_{K_{2}\left(y_{0}\right)} z_{0}^{2} \in K_{2}\left(y_{0}\right), \omega_{0}^{1} \in B_{1}\left(x_{0}, y_{0}\right), \omega_{0}^{2} \in B_{2}\left(x_{0}, y_{0}\right)$. We compute $\left\{x_{n}\right\},\left\{y_{n}\right\},\left\{z_{n}^{1}\right\},\left\{z_{n}^{2}\right\},\left\{\omega_{n}^{1}\right\}$ and $\left\{\omega_{n}^{2}\right\}$ by the following iterative schemes:

$$
\begin{aligned}
& x_{n}=P_{K_{1}\left(x_{n}\right)} z_{n}^{1}, \\
& y_{n}=P_{K_{2}\left(y_{n}\right)} z_{n}^{2}, \\
& z_{n+1}^{1}=x_{n}-\rho_{1}\left(A_{1}\left(x_{n}, y_{n}\right)+\omega_{n}^{1}\right), \\
& z_{n+1}^{2}=y_{n}-\rho_{2}\left(A_{2}\left(x_{n}, y_{n}\right)+\omega_{n}^{2}\right), \\
& \omega_{n}^{1} \in B_{1}\left(x_{n}, y_{n}\right), \quad\left\|\omega_{n+1}^{1}-\omega_{n}^{1}\right\| \leq\left(1+\frac{1}{n+1}\right) H\left(B_{1}\left(x_{n+1}, y_{n+1}\right), B_{1}\left(x_{n}, y_{n}\right)\right), \\
& \omega_{n}^{2} \in B_{2}\left(x_{n}, y_{n}\right), \quad\left\|\omega_{n+1}^{2}-\omega_{n}^{2}\right\| \leq\left(1+\frac{1}{n+1}\right) H\left(B_{2}\left(x_{n+1}, y_{n+1}\right), B_{2}\left(x_{n}, y_{n}\right)\right),
\end{aligned}
$$

where $n=0,1,2, \ldots$

If $K_{1}(x)=K_{2}(y)=K$, we have the following iterative algorithm from Algorithm 4.3. 
Algorithm 4.4 For any given $\left(z_{0}^{1}, z_{0}^{2}\right) \in H \times H$, such that $x_{0}=P_{K} z_{0}^{1} \in K, y_{0}=P_{K} z_{0}^{2} \in K$, $\omega_{0}^{1} \in B_{1}\left(x_{0}, y_{0}\right), \omega_{0}^{2} \in B_{2}\left(x_{0}, y_{0}\right)$, we compute $\left\{x_{n}\right\},\left\{y_{n}\right\},\left\{z_{n}^{1}\right\},\left\{z_{n}^{2}\right\},\left\{\omega_{n}^{1}\right\}$ and $\left\{\omega_{n}^{2}\right\}$ by the following iterative schemes:

$$
\begin{aligned}
& x_{n}=P_{K} z_{n}^{1}, \\
& y_{n}=P_{K} z_{n}^{2}, \\
& z_{n+1}^{1}=x_{n}-\rho_{1}\left(A_{1}\left(x_{n}, y_{n}\right)+\omega_{n}^{1}\right), \\
& z_{n+1}^{2}=y_{n}-\rho_{2}\left(A_{2}\left(x_{n}, y_{n}\right)+\omega_{n}^{2}\right), \\
& \omega_{n}^{1} \in B_{1}\left(x_{n}, y_{n}\right), \quad\left\|\omega_{n+1}^{1}-\omega_{n}^{1}\right\| \leq\left(1+\frac{1}{n+1}\right) H\left(B_{1}\left(x_{n+1}, y_{n+1}\right), B_{1}\left(x_{n}, y_{n}\right)\right), \\
& \omega_{n}^{2} \in B_{2}\left(x_{n}, y_{n}\right), \quad\left\|\omega_{n+1}^{2}-\omega_{n}^{2}\right\| \leq\left(1+\frac{1}{n+1}\right) H\left(B_{2}\left(x_{n+1}, y_{n+1}\right), B_{2}\left(x_{n}, y_{n}\right)\right),
\end{aligned}
$$

where $n=0,1,2, \ldots$

Theorem 4.5 Let $H$ be a real Hilbert space, $K_{1}(x)$ and $K_{2}(y)$ be two nonempty closed convex sets in $H$. For $i=1,2$, let nonlinear mappings $A_{i}: K_{1}(x) \times K_{2}(y) \rightarrow H$ be $\left(\xi_{i}, \eta_{i}\right)$-Lipschitz continuous and $\left(\tau_{i}, \zeta_{i}\right)$-relaxed co-coercive with respect to the ith argument, $B_{i}: H \times H \rightarrow$ $C(H)$ be $\left(\alpha_{i}, \beta_{i}\right)$-H-Lipschitz continuous, if Assumption 2.4 holds and there exist constants $\rho_{i}>0$ such that

$$
\begin{array}{r}
0<\max \left\{\frac{1}{1-s}\left[\sqrt{1+2 \rho_{1}\left(\tau_{1} \xi_{1}^{2}-\zeta_{1}\right)+\rho_{1}^{2} \xi_{1}^{2}}+\rho_{1} \alpha_{1}+\rho_{2}\left(\alpha_{2}+\xi_{2}\right)\right],\right. \\
\left.\frac{1}{1-s}\left[\sqrt{1+2 \rho_{2}\left(\tau_{2} \eta_{2}^{2}-\zeta_{2}\right)+\rho_{2}^{2} \eta_{2}^{2}}+\rho_{2} \beta_{2}+\rho_{1}\left(\beta_{1}+\eta_{1}\right)\right]\right\}<1,
\end{array}
$$

then there exist $\left(x, y, \omega_{1}, \omega_{2}, z_{1}, z_{2}\right)$ satisfying the system of generalized implicit Wiener-Hopf equations (4.1). So, the problem (1.2) admits solutions ( $\left.x, y, \omega_{1}, \omega_{2}\right)$ and sequences $\left\{x_{n}\right\},\left\{y_{n}\right\}$, $\left\{z_{n}^{1}\right\},\left\{z_{n}^{2}\right\},\left\{\omega_{n}^{1}\right\}$ and $\left\{\omega_{n}^{2}\right\}$ which are generated by Algorithm 4.3 converge to $x, y, z_{1}, z_{2}, \omega_{1}$ and $\omega_{2}$ respectively.

Proof By (4.8), we have

$$
\begin{aligned}
\left\|z_{n+1}^{1}-z_{n}^{1}\right\|= & \left\|x_{n}-\rho_{1}\left(A_{1}\left(x_{n}, y_{n}\right)+\omega_{n}^{1}\right)-\left[x_{n-1}-\rho_{1}\left(A_{1}\left(x_{n-1}, y_{n-1}\right)+\omega_{n-1}^{1}\right)\right]\right\| \\
\leq & \left\|x_{n}-x_{n-1}-\rho_{1}\left(A_{1}\left(x_{n}, y_{n}\right)-A_{1}\left(x_{n-1}, y_{n}\right)\right)\right\| \\
& +\rho_{1}\left[\left\|\omega_{n}^{1}-\omega_{n-1}^{1}\right\|+\left\|A_{1}\left(x_{n-1}, y_{n}\right)-A_{1}\left(x_{n-1}, y_{n-1}\right)\right\|\right] .
\end{aligned}
$$

Since $A_{1}$ is $\left(\tau_{1}, \zeta_{1}\right)$-relaxed co-coercive with respect to the first argument and Lipschitz continuous, we have

$$
\begin{aligned}
& \left\|x_{n}-x_{n-1}-\rho_{1}\left(A_{1}\left(x_{n}, y_{n}\right)-A_{1}\left(x_{n-1}, y_{n}\right)\right)\right\|^{2} \\
& =\left\|x_{n}-x_{n-1}\right\|^{2}-2 \rho_{1}\left\langle A_{1}\left(x_{n}, y_{n}\right)-A_{1}\left(x_{n-1}, y_{n}\right), x_{n}-x_{n-1}\right\rangle \\
& \quad+\rho_{1}^{2}\left\|A_{1}\left(x_{n}, y_{n}\right)-A_{1}\left(x_{n-1}, y_{n}\right)\right\|^{2}
\end{aligned}
$$




$$
\begin{aligned}
\leq & \left\|x_{n}-x_{n-1}\right\|^{2}-2 \rho_{1}\left[\left(-\tau_{1}\right)\left\|A_{1}\left(x_{n}, y_{n}\right)-A_{1}\left(x_{n-1}, y_{n}\right)\right\|^{2}+\zeta_{1}\left\|x_{n}-x_{n-1}\right\|^{2}\right] \\
& +\rho_{1}^{2} \xi_{1}^{2}\left\|x_{n}-x_{n-1}\right\|^{2} \\
\leq & {\left[1+2 \rho_{1}\left(\tau_{1} \xi_{1}^{2}-\zeta_{1}\right)+\rho_{1}^{2} \xi_{1}^{2}\right]\left\|x_{n}-x_{n-1}\right\|^{2}, }
\end{aligned}
$$

and

$$
\left\|A_{1}\left(x_{n-1}, y_{n}\right)-A_{1}\left(x_{n-1}, y_{n-1}\right)\right\| \leq \eta_{1}\left\|y_{n}-y_{n-1}\right\| .
$$

From $\left(\alpha_{1}, \beta_{1}\right)$ - $H$-Lipschitz continuity of $B_{1}$ and (4.10), we have

$$
\begin{aligned}
\left\|\omega_{n}^{1}-\omega_{n-1}^{1}\right\| & \leq\left(1+\frac{1}{n}\right) H\left(B_{1}\left(x_{n}, y_{n}\right), B_{1}\left(x_{n-1}, y_{n-1}\right)\right) \\
& \leq\left(1+\frac{1}{n}\right)\left[\alpha_{1}\left\|x_{n}-x_{n-1}\right\|+\beta_{1}\left\|y_{n}-y_{n-1}\right\|\right] .
\end{aligned}
$$

Combining (4.13), (4.14), (4.15) and (4.16), we obtain

$$
\begin{aligned}
\left\|z_{n+1}^{1}-z_{n}^{1}\right\| \leq & {\left[\sqrt{1+2 \rho_{1}\left(\tau_{1} \xi_{1}^{2}-\zeta_{1}\right)+\rho_{1}^{2} \xi_{1}^{2}}+\rho_{1}\left(1+\frac{1}{n}\right) \alpha_{1}\right]\left\|x_{n}-x_{n-1}\right\| } \\
& +\rho_{1}\left[\left(1+\frac{1}{n}\right) \beta_{1}+\eta_{1}\right]\left\|y_{n}-y_{n-1}\right\| .
\end{aligned}
$$

Similarly, we can have

$$
\begin{aligned}
\left\|z_{n+1}^{2}-z_{n}^{2}\right\| \leq & \rho_{2}\left[\left(1+\frac{1}{n}\right) \alpha_{2}+\xi_{2}\right]\left\|x_{n}-x_{n-1}\right\| \\
+ & {\left[\sqrt{1+2 \rho_{2}\left(\tau_{2} \eta_{2}^{2}-\zeta_{2}\right)+\rho_{2}^{2} \eta_{2}^{2}}+\rho_{2}\left(1+\frac{1}{n}\right) \beta_{2}\right]\left\|y_{n}-y_{n-1}\right\| . }
\end{aligned}
$$

By (4.6), Lemma 2.3 and Assumption 2.4,

$$
\begin{aligned}
\left\|x_{n}-x_{n-1}\right\|= & \left\|P_{K_{1}\left(x_{n}\right)} z_{n}^{1}-P_{K_{1}\left(x_{n-1}\right)} z_{n-1}^{1}\right\| \\
\leq & \left\|P_{K_{1}\left(x_{n}\right)} z_{n}^{1}-P_{K_{1}\left(x_{n-1}\right)} z_{n}^{1}\right\| \\
& \quad+\left\|P_{K_{1}\left(x_{n-1}\right)} z_{n}^{1}-P_{K_{1}\left(x_{n-1}\right)} z_{n-1}^{1}\right\| \\
\leq & S_{1}\left\|x_{n}-x_{n-1}\right\|+\left\|z_{n}^{1}-z_{n-1}^{1}\right\|,
\end{aligned}
$$

which implies that

$$
\left\|x_{n}-x_{n-1}\right\| \leq \frac{1}{1-s_{1}}\left\|z_{n}^{1}-z_{n-1}^{1}\right\|
$$

Similarly, we can obtain

$$
\left\|y_{n}-y_{n-1}\right\| \leq \frac{1}{1-s_{2}}\left\|z_{n}^{2}-z_{n-1}^{2}\right\|
$$


By (4.17)-(4.20), we have

$$
\begin{aligned}
& \left\|z_{n+1}^{1}-z_{n}^{1}\right\|+\left\|z_{n+1}^{2}-z_{n}^{2}\right\| \\
& \leq \frac{1}{1-s_{1}}\left\{\sqrt{1+2 \rho_{1}\left(\tau_{1} \xi_{1}^{2}-\zeta_{1}\right)+\rho_{1}^{2} \xi_{1}^{2}}+\rho_{1}\left(1+\frac{1}{n}\right) \alpha_{1}+\rho_{2}\left[\left(1+\frac{1}{n}\right) \alpha_{2}+\xi_{2}\right]\right\} \\
& \quad \times\left\|z_{n}^{1}-z_{n-1}^{1}\right\| \\
& \quad+\frac{1}{1-s_{2}}\left\{\sqrt{1+2 \rho_{2}\left(\tau_{2} \eta_{2}^{2}-\zeta_{2}\right)+\rho_{2}^{2} \eta_{2}^{2}}+\rho_{2}\left(1+\frac{1}{n}\right) \beta_{2}+\rho_{1}\left[\left(1+\frac{1}{n}\right) \beta_{1}+\eta_{1}\right]\right\} \\
& \quad \times\left\|z_{n}^{2}-z_{n-1}^{2}\right\| \\
& \leq \theta_{n}\left(\left\|z_{n}^{1}-z_{n-1}^{1}\right\|+\left\|z_{n}^{2}-z_{n-1}^{2}\right\|\right),
\end{aligned}
$$

where

$$
\begin{aligned}
\theta_{n}= & \max \left\{\frac{1}{1-s_{1}}\left[\sqrt{1+2 \rho_{1}\left(\tau_{1} \xi_{1}^{2}-\zeta_{1}\right)+\rho_{1}^{2} \xi_{1}^{2}}+\rho_{1}\left(1+\frac{1}{n}\right) \alpha_{1}+\rho_{2}\left(\left(1+\frac{1}{n}\right) \alpha_{2}+\xi_{2}\right)\right],\right. \\
& \left.\frac{1}{1-s_{2}}\left[\sqrt{1+2 \rho_{2}\left(\tau_{2} \eta_{2}^{2}-\zeta_{2}\right)+\rho_{2}^{2} \eta_{2}^{2}}+\rho_{2}\left(1+\frac{1}{n}\right) \beta_{2}+\rho_{1}\left(\left(1+\frac{1}{n}\right) \beta_{1}+\eta_{1}\right)\right]\right\} .
\end{aligned}
$$

Let

$$
\begin{aligned}
\theta= & \max \left\{\frac{1}{1-s_{1}}\left[\sqrt{1+2 \rho_{1}\left(\tau_{1} \xi_{1}^{2}-\zeta_{1}\right)+\rho_{1}^{2} \xi_{1}^{2}}+\rho_{1} \alpha_{1}+\rho_{2}\left(\alpha_{2}+\xi_{2}\right)\right],\right. \\
& \left.\frac{1}{1-s_{2}}\left[\sqrt{1+2 \rho_{2}\left(\tau_{2} \eta_{2}^{2}-\zeta_{2}\right)+\rho_{2}^{2} \eta_{2}^{2}}+\rho_{2} \beta_{2}+\rho_{1}\left(\beta_{1}+\eta_{1}\right)\right]\right\},
\end{aligned}
$$

then $\theta_{n} \rightarrow \theta$ as $n \rightarrow \infty$. By (4.12), we know that $0<\theta<1$. So, (4.21) implies that $\left\{z_{n}^{1}\right\}$ and $\left\{z_{n}^{2}\right\}$ are both Cauchy sequences. By (4.19) and (4.20), we know that $\left\{x_{n}\right\}$ and $\left\{y_{n}\right\}$ are both Cauchy sequences respectively. So, there exist $\left(z_{1}, z_{2}\right) \in H \times H$ and $(x, y) \in K_{1}(x) \times$ $K_{2}(y)$ such that $z_{n}^{1} \rightarrow z_{1}, z_{n}^{2} \rightarrow z_{2}, x_{n} \rightarrow x$ and $y_{n} \rightarrow y$ as $n \rightarrow \infty$. In a similar way as in Theorem 3.3, we know $\left\{\omega_{n}^{1}\right\}$ and $\left\{\omega_{n}^{2}\right\}$ are also Cauchy sequences and there exist $\omega_{1} \in$ $B_{1}(x, y)$ and $\omega_{2} \in B_{2}(x, y)$ such that $\omega_{n}^{1} \rightarrow \omega_{1}$ and $\omega_{n}^{2} \rightarrow \omega_{2}$.

By the continuity of the mappings $A_{1}, A_{2}, B_{1}, B_{2}, P_{K_{1}(x)}, P_{K_{2}(y)}$ and Algorithm 4.3, as $n \rightarrow \infty$, we have

$$
\left\{\begin{array}{l}
x=P_{K_{1}(x)} z_{1}, \\
y=P_{K_{2}(y)} z_{2}, \\
z_{1}=x-\rho_{1}\left(A_{1}(x, y)+\omega_{1}\right), \\
z_{2}=y-\rho_{2}\left(A_{2}(x, y)+\omega_{2}\right),
\end{array}\right.
$$

where $\rho_{1}>0, \rho_{2}>0$ are constants. That is just (4.4). By Lemma 4.1, we know that $\left(x, y, z_{1}, z_{2}, \omega_{1}, \omega_{2}\right)$ satisfy the generalized implicit Wiener-Hopf equations (4.1). So, we claim that $\left(x, y, \omega_{1}, \omega_{2}\right)$ is a solution of the problem (1.2). This completes the proof.

If $K_{1}(x)=K_{2}(y)=K$, we do not need Assumption 2.4 and we can obtain the following theorem from Theorem 4.5 . 
Theorem 4.6 Let $H$ be a real Hilbert space, $K$ be a nonempty closed convex set in $H$. For $i=1,2$, let nonlinear mappings $A_{i}: K \times K \rightarrow H$ be $\left(\xi_{i}, \eta_{i}\right)$-Lipschitz continuous and $\left(\tau_{i}, \zeta_{i}\right)$-relaxed co-coercive with respect to the ith argument, $B_{i}: H \times H \rightarrow C(H)$ be $\left(\alpha_{i}, \beta_{i}\right)$ $H$-Lipschitz continuous if there exist constants $\rho_{i}>0$ such that

$$
\begin{array}{r}
0<\max \left\{\sqrt{1+2 \rho_{1}\left(\tau_{1} \xi_{1}^{2}-\zeta_{1}\right)+\rho_{1}^{2} \xi_{1}^{2}}+\rho_{1} \alpha_{1}+\rho_{2}\left(\alpha_{2}+\xi_{2}\right),\right. \\
\left.\quad \sqrt{1+2 \rho_{2}\left(\tau_{2} \eta_{2}^{2}-\zeta_{2}\right)+\rho_{2}^{2} \eta_{2}^{2}}+\rho_{2} \beta_{2}+\rho_{1}\left(\beta_{1}+\eta_{1}\right)\right\}<1,
\end{array}
$$

then there exist $\left(x, y, \omega_{1}, \omega_{2}, z_{1}, z_{2}\right)$ satisfying (4.5). So, the generalized Wiener-Hopf equations (4.2) and the problem (1.1) admit the same solutions $\left(x, y, \omega_{1}, \omega_{2}\right)$ and sequences $\left\{x_{n}\right\}$, $\left\{y_{n}\right\},\left\{z_{n}^{1}\right\},\left\{z_{n}^{2}\right\},\left\{\omega_{n}^{1}\right\}$ and $\left\{\omega_{n}^{2}\right\}$ which are generated by Algorithm 4.4 converge to $x, y, z_{1}, z_{2}$, $\omega_{1}$ and $\omega_{2}$ respectively.

Remark 4.7 It is the first time that the system of generalized Wiener-Hopf equations technique has been used to solve the system of generalized variational inequalities problem. And for a suitable and appropriate choice of the mappings $A_{i}, B_{i}$ and $K_{i}$, Theorem 3.3 and Theorem 4.5 include many important known results of variational inequality as special cases.

Remark 4.8 It is easy to see that a $\gamma$-strongly monotone mapping must be a $(\tau, \zeta)$-relaxed co-coercive mapping, whenever $\tau=0, \zeta=\gamma$. Therefore, the class of the $(\tau, \zeta)$-relaxed cocoercive mappings is a more general one. Hence, the results presented in the paper include many known results as special cases.

Competing interests

The authors declare that they have no competing interests.

Authors' contributions

The work presented here was carried out in collaboration between all authors. All authors read and approve the final manuscript.

\section{Author details}

'College of Applied Science, Jiangxi University of Science and Technology, Ganzhou, Jiangxi 341000, P.R. China.

${ }^{2}$ Department of science, Nanchang Institute of Technology, Nanchang, 330099, P.R. China.

\section{Acknowledgements}

The work is supported by the National Natural Science Foundation of China (61064006), the Natural Science Foundation of Jiangxi Province (No.2009GZS0009) and the Educational Research Project of Jiangxi Province (GJJ09249).

Received: 24 August 2012 Accepted: 14 December 2012 Published: 8 January 2013

\section{References}

1. Noor, MA: Generalized set-valued mixed nonlinear quasi variational inequalities. Korean J. Comput. Appl. Math. 5(1), 73-89 (1998)

2. Noor, MA: Some developments in general variational inequalities. Appl. Math. Comput. 152(1), $199-277$ (2004)

3. Glowinski, R, Lions, JJ, Tremolieres, R: Numerical Analysis of Variational Inequalities. North-Holland, Amsterdam (1981)

4. Huang, NJ: Generalized nonlinear variational inclusions with noncompact valued mappings. Appl. Math. Lett. 9(3), 25-29 (1996)

5. Zhong, RY, Huang, NJ: Strict feasibility for generalized mixed variational inequality in reflexive Banach spaces. J. Optim. Theory Appl. 152, 696-709 (2012)

6. Qiu, YQ, Liu, LW: A new system of generalized quasi-variational-like inclusion in Hilbert spaces. Comput. Math. Appl. 59(1), 1-8 (2010)

7. Ding, XP: Existence and algorithm of solutions for generalized mixed implicit quasi-variational inequalities. Appl. Math. Comput. 113(1), 67-80 (2000)

8. Verma, RU: Projection methods, algorithms, and a new system of nonlinear variational inequalities. Comput. Math. Appl. 41(7-8), 1025-1031 (2001) 
9. Xia, FQ, Huang, NJ: A projection-proximal point algorithm for solving generalized variational inequalities. J. Optim. Theory Appl. 150, 98-117 (2011)

10. Chang, SS: Set-valued variational inclusions in Banach spaces. J. Math. Anal. Appl. 248(2), 438-454 (2000)

11. Wang, SH, Huang, NJ, ORegan, D: Well-posedness for generalized quasi-variational inclusion problems and for optimization problems with constraints. J. Glob. Optim. (2012). doi:10.1007/s10898-012-9980-6

12. Noor, MA: Projection iterative methods for extended general variational inequalities. J. Appl. Math. Comput. 32(1) 83-95 (2010)

13. Petrot, N: Existence and algorithm of solutions for general set-valued Noor variational inequalities with relaxed $(\mu, v)$-cocoercive operators in Hilbert spaces. J. Appl. Math. Comput. 32(2), 393-404 (2010)

doi:10.1186/1687-1812-2013-4

Cite this article as: Qiu and Li: The existence of solutions for systems of generalized set-valued nonlinear quasi-variational inequalities. Fixed Point Theory and Applications 2013 2013:4.

Submit your manuscript to a SpringerOpen ${ }^{\odot}$ journal and benefit from:

- Convenient online submission

Rigorous peer review

- Immediate publication on acceptance

- Open access: articles freely available online

- High visibility within the field

- Retaining the copyright to your article 